\title{
Setting Objective Clinical Assessment Tools for Circadian Rhythm Sleep-Wake Disorders - A Community-Based Cross-Sectional Epidemiological Study
}

Frangiskos Frangopoulos' Ivi Nicolaou'

Savvas Zannetos (D) ${ }^{2}$

Nicholas-Tiberio Economou ${ }^{3}$

Tonia Adamide III'

Andreas Georgiou'

Pantelis Theodoros Nikolaidis (D) ${ }^{4}$

Thomas Rosemann $\mathbb{1 D}^{5}$

Beat Knechtle ${ }^{5}$

Georgia Trakada $\mathbb{D}^{3}$

'Respiratory Department, Nicosia General Hospital, Nicosia, Cyprus; ${ }^{2}$ Health Economics and Statistics, Neapolis University, Paphos, Cyprus; ${ }^{3}$ Division of Pulmonology, Department of Clinical Therapeutics, National and Kapodistrian University of Athens, School of Medicine, Alexandra Hospital, Athens, Greece; ${ }^{4}$ School of Health and Caring Sciences, University of West Attica, Athens, Greece; ${ }^{5}$ Institute of Primary Care, University of Zurich, Zurich, Switzerland
Correspondence: Georgia Trakada Department of Clinical Therapeutics, National and Kapodistrian University of Athens, School of Medicine, Alexandra Hospital, Vas Sofias 80, Athens, II528, Greece

Tel +302103381488

$\mathrm{Fax}+302132162889$

Email gtrakada@hotmail.com
Introduction: Circadian rhythm sleep-wake disorder (CRSWD) is an often-misdiagnosed group of sleep disturbances with limited data concerning diagnostic and therapeutic algorithms in the general population. Therefore, reported prevalence varies in the literature due to different case definitions, methodological, and environmental factors.

Objectives: The purpose of our cross-sectional study was to estimate the prevalence of CRSWD in the general population of Cyprus and to suggest clinical parameters for the assessment of atypical sleep schedules. The estimation was carried out by introducing normal preset sleep time limits, according to the imposed local environment and by administering well-established questionnaires for symptoms and consequence dysfunction, as objective evaluation tools.

Methods: In a nationwide epidemiological survey, 4118 Cypriot adult participants, selected and stratified to represent the general population, were interviewed on sleep habits and complaints by computer-assisted telephone interviewing (CATI). In the second stage, 250 adults were randomly selected from the initial representative sample, for a CRSWD assessment. According to the proposed diagnostic criteria, patients were interviewed about sleepiness and/or insomnia, and daytime dysfunction (fatigue, anxiety, and depression). They were also assessed by a weekly sleep diary for sleep schedules. Finally, all participants underwent a Type III Sleep Study, to rule out obstructive sleep apnea.

Results: From 195 enrolled participants (response rate 78\%), 25 individuals (12.8\%) met the criteria for CRSWD. The two most prevalent disorders were delayed sleep-wake phase disorder (DSWPD) (10pts, 5.1\%) and shift work sleep disorder (SWD) (13, 6.7\%). Less prevalent disorders included irregular sleep-wake rhythm disorder (ISWRD) $(1,0.5 \%)$ and advanced sleep-wake phase disorder (ASWPD) (1,0.5\%).

Conclusions: According to our data, CRSWDs are common in the general population of Cyprus, especially DSWPD and SWD. Affected individuals usually suffer from sleep deprivation and complain about insomnia, sleepiness and depression.

Keywords: circadian sleep wake phase disorders, prevalence, sleep deprivation, guidelines

\section{Introduction}

Sleep-wake cycle is regulated by a homeostatic drive that promotes an increased propensity towards sleep at night and an endogenous, circadian drive that facilitates wakefulness during the day. Circadian rhythm is self-sustaining with a periodicity of approximately 24 hours, however external factors such as light, working 
schedules, social life, exercise, and mealtimes can affect duration, quality and appropriately timed sleep. The internal biological clock is stationed in the suprachiasmatic nucleus. It maintains rhythmicity of the activity-rest cycle at a genetic level through a complex transcriptiontranslation feedback loop, regulated by clock genes and modulated by exogenous factors called synchronizers. ${ }^{1,2}$

CRSWDs represent a misalignment between the individual's sleep schedule and that which is desired or considered normal by the society. They emerge from deregulation of the inner circadian pacemaker or the desynchronization of the endogenous system with the external 24-hour time cues or zeitgebers (German for time givers). ${ }^{3,4}$

Weitzman et al first recognized delayed sleep phase syndrome (DSPS) to $7 \%$ of their insomnia patients. ${ }^{5}$ Later on, the American Sleep Disorders Association recognized a separate, major sleep nosology, the circadian rhythm sleep disorders (CRSD). ${ }^{6}$ The definition and diagnosis criteria for CRSWDs are now set by the American Academy of Sleep Medicine (AASM) in the ICSD-3.,

CRSWDs are predominantly caused by innate phenomena, with a varied contribution of exogenous factors. The underlying problem is that sleep episodes and wake intervals occur untimely at undesired times. Consequently, when obliged to comply with a normal societal sleepwake routine, individuals may complain about symptoms of insomnia or excessive daytime sleepiness.

Insufficient epidemiological data exist for CRSWD in the general population. These data are probably under or misdiagnosed because of coexistence with other sleep disorders causing sleepiness or insomnia, mental or physical disorders masking CRSWD, lack of acknowledgement from the medical professionals, community ignorance, and misperception as to what is considered normal nowadays.

Data from South Europe are missing. Previous studies in the general population were conducted 20-30 years ago in Japan and Norway and since then, lifestyle has changed dramatically. ${ }^{9,10}$ There is also a general lack of evidence to support current international guidelines concerning clinical evaluation, diagnosis and treatment of these sleep disorders.

We designed a community-based cross-sectional study in order to estimate the prevalence of CRSWD in the general population of Cyprus. A second objective of our study was to introduce some practical suggestions for the assessment of atypical sleep schedules in relation to the usual/acceptable timetable, mainly by defining what is considered an ordinary sleep time schedule in the general population, as it is framed by the local environmental, societal and economic conditions. This would allow presetting cut-off time limits for certain CRSWDs. In order to avoid subjectivity and increase the validity of clinical diagnosis, we introduced the application of wellestablished questionnaires for symptoms and consequence dysfunction in the diagnostic procedure.

\section{Participants and Methods Study Population}

A stratified random sample survey of sleep complaints by telephone recruiting was conducted in the adult general population, as previously described, to estimate the prevalence of Sleep Disorders in Cyprus. ${ }^{11}$ The inclusion criteria were: 1) age $\geq 18,2$ ) permanent inhabitants of Cyprus, 3) consent to participate in the survey. The sample was stratified according to the last demographic report (2016) by district, rural or urban area, gender and age. The survey was conducted by computer-assisted telephone interviewing (CATI) method.

A secondary cross-sectional nationwide epidemiological survey was piloted to examine the prevalence of circadian rhythm disorders in the adult population.

From the initial representative sample of the Cypriot adult population (4118 participants), 250 adults were randomly selected to participate in the second-stage procedure. Non-strict inclusion or exclusion criteria were applied in order to guarantee unbiased selection, minimize the necessary sample size and achieve reliable results.

This study was conducted in accordance with the Declaration of Helsinki. The ethical committees of both the General Hospital in Nicosia, Cyprus and "Alexandra" University Hospital, in Greece, approved the study protocol. All subjects gave consent to participate in the study after appropriate information was given.

\section{Study Protocol}

All participants answered a series of questionnaires to assess major symptoms as follows: 1) Epworth Sleepiness Scale (ESS) to evaluate sleepiness, and 2) Athens Insomnia Scale (AIS) to evaluate symptoms of insomnia. The negative association between sleep-wake schedule and functionality or status was assessed for fatigue (by Fatigue Severity Scale, FSS), depression (by Beck 
Depression Inventory, BDI-II) and anxiety/depression (by Hospital Anxiety and Depression Scale, HADS).

The ESS evaluates the tendency to fall asleep in eight daily situations. The ESS score ranges from 0 to 24 , and a score $\geq 10$ indicates excessive daytime sleepiness. ${ }^{12}$ AIS assesses five factors related to nocturnal sleep and three elements related to daytime dysfunction. ${ }^{13} \mathrm{~A}$ cutoff score of $\geq 6$ on the AIS is used to establish the diagnosis of insomnia. FSS estimates the fatigue severity in different situations of the previous week, by using nine questions. The final score is the mean value of the nine items, and a score $\geq 4$ is interpreted as fatigue. ${ }^{14}$ BDI-II includes 21 questions. ${ }^{15}$ Higher total scores indicate more severe depressive symptoms. HADS is a 14item depression and anxiety screening tool, assessing the severity of symptoms in medically ill patients, while excluding somatic symptoms potentially attributable to comorbid medical conditions. ${ }^{16}$ Each question is scored from 0 to 3, with a total score up to 21 for each subscale. All participants also completed a seven-day diary of sleeping and waking times and other related information (naps, medications, exercise, caffeine consumption, etc.).

Age, food, alcohol, caffeine, exercise, medication, noise, individuals' susceptibility to noise, marital status, children, coexistence of other types of sleep disturbances, nocturia or other reasons for systematic awakenings, sleep deprivation, working and social schedule, can alter the individual's circadian clock. However, there is no feasible way to eliminate all the confounding variables and stratify the sample.

Finally, all participants underwent a Type III Sleep Study, to rule out obstructive sleep apnea (OSA). A cutoff score of $\geq 15$ respiratory events per hour of monitoring time satisfies the criteria for OSA, even in the absence of associated symptoms. The Type III sleep study is ambulatory performed with limited channel devices. The equipment used in the study (Embletta MPR Sleep Systems by Natus and Remlogic software), comprise 6 monitoring channels (nasal pressure cannula, inductance plethysmography for thorax and abdomen, heart rate, oxygen saturation, body position detection). ${ }^{17}$

\section{Diagnostic Criteria}

Diagnostic criteria for CRSWD were in accordance with International Classification of Sleep Disorders ${ }^{7,8}$ and selected subjective methods for scoring and supporting the diagnosis.
The general categorization criteria were: 1) sleepiness and/or symptoms of insomnia, according to ESS and AIS scores and 2) negative association between sleep-wake schedule and functionality or status (mental, physical, social, professional or academic impairment), according to FSS, BDI-II and HADS. However, the assessment of daytime consequences is challenging, especially when attempting a sole attribution to the investigated condition.

A specific criterion for each category's subtype was added, as stated below:

1) Time Zone Change Syndrome or jet lag disorder (JLD): Long-distance air traveling in previous days is the specific situation that causes a temporary mismatch between the timing of the sleep and wake cycle generated by a change in time zone.

2) Shift work sleep disorder (SWD): Working in shifts (night, early morning or rotating shifts) is the specific situation that overlaps with all or a percentage of conventional night-time sleep periods.

3) Irregular sleep-wake rhythm disorder (ISWRD): It is characterized by multiple (at least 3) sleep-wake phase changes (1-4 hours each phase) within a 24-hour period.

4) Non-24-h sleep-wake rhythm disorder or free-running disorder (N24SWD): It is characterized by 1-2 hours daily displacement of the time a person sleeps and wakes up, making it impossible to maintain a steady sleep-wake schedule.

5) Advanced sleep-wake phase disorder (ASWPD): Sleep and wake-up times are advanced by $2-3$ hours, contrasting the desired or socially acceptable timetable Patients typically sleep between 6 and 8pm, but not after $9 \mathrm{pm}$ and wake up before $5 \mathrm{am}$. Sleep onset $\leq 9 \mathrm{pm}$ and sleep offset $\leq 5 \mathrm{am}$ were set as thresholds for the diagnosis.

6) Delayed sleep-wake phase disorder (DSWPD): There is a persistent phase delay in the major sleep period compared to conventional or desired sleep times, by 3-6 hours, accompanied by a complaint of inability to fall asleep at the desired clock time. Usually, patients sleep after midnight and wake up late in the morning, unless obliged otherwise (early morning wake up). The specific criterion selected is falling asleep between 12am and 3am.

7) Circadian sleep-wake disorder not otherwise specified (residual).

According to the ICD-3 and the study protocol, the general categorization criteria were: 1) excessive daytime sleepiness (ESS $>10)$, and/or symptoms of insomnia (AIS $\geq 6$ ) and 2) negative association between sleep-wake schedule and functionality or status (mental, physical, social impairment). The impairment was assessed with 
one or more positive questionnaires: FSS $(\geq 4)$, BDI-II $(\geq 14)$ and HADS $(\geq 8)$. Participants with an Apnea Hypopnea Index $\geq 15$ were excluded from having CRSWD as major sleep symptoms; therefore, daily dysfunction and mood disturbances could be fairly attributed to moderate/severe OSA.

The third criterion was specific for each category's subtype: 1) Long-distance air traveling in previous days for JLD, 2) Working in shifts for SWD, 3) For ISWRD at least 3 sleep-wake phase changes (1-4 hours each phase) within a 24-hour period 4) Daily displacement for 1-2 hours of the sleep-wake phases, for N24SWD, 5) Sleep onset $\leq 9 \mathrm{pm}$ and sleep offset $\leq 5 \mathrm{am}$ as thresholds for the diagnosis of ASWPD and 6) Falling asleep between 12am and 3am for DSWPD.

The two general criteria (symptoms and sequel dysfunction) and the specific criterion set for each subcategory (eg, shift work for SWD) should be met for the diagnosis of a CRSWD.

However, the chronic deregulation of sleep-wake pattern for $\geq 3$ months, as a general criterion for the diagnosis, cannot be applied to every CRSWD. For example, JLD has short endurance. Also, in order to diagnose a circadian rhythm sleep disorder, another general criterion states that the sleep disturbance should not be better explained by another current sleep disorder, medical, neurological, or mental disease, and use of medications or substances affecting sleep. A detailed, medical history can exclude several confounding factors. However, it is a complicated task. For example, in DSWPD, excessive use of caffeine may facilitate compliance with a normal societal sleepwake routine and not disturbed sleep.

\section{Statistical Analysis}

Statistical analysis included summarization of the data in tables and charts and it was performed by using the IBM SPSS Statistics v.25 program. Descriptive statistics such as means and standard deviations for continuous variables as well as counts and percentages for nominal variables were used to examine characteristics for all participants. Hypothesis testing was performed in order to accept or not the null hypothesis. Specifically, $\chi 2$ test was performed for comparing nominal variables, while $t$-test was used to compare continuous variables. All tests were two-sided and the level of significance was set to $\alpha=0.05$; thus, a p-value lower than 0.05 was considered statistically significant.
Continuous data did not deviate significantly from normality. No extreme cases were identified as well as no heavy tails on the q-q plot were observed. Furthermore, for all parametric tests reported, the equivalent non-parametric test was also performed to observe if there is any difference in statistical significance. No such difference was observed; thus, due to the large sample size as well as the agreement on the test conclusion of parametric and non-parametric tests, the parametric test and its subsequent $\mathrm{p}$ values were reported.

\section{Results}

From 4118 eligible responders, stratified to represent the Cypriot population, a cohort of 250 individuals - randomly selected by SPSS - was enrolled. One hundred ninety-five (195) individuals accepted to participate, yielding a margin of error of $\pm 1.53 \%$, at a Confidence Level of $95 \%$. The response rate was $78 \%$. All, 128 males $(65.6 \%)$ and 67 females $(34.4 \%)$, with mean age $54.91 \pm 13.29$ years old, answered the questionnaires, completed a seven-day diary and underwent the sleep study.

\section{Sleep Characteristics in the General Population}

Subjective sleep time of $7-8$ h was reported by $38.6 \%$ of the study population, $6-7 \mathrm{~h} 34 \%$, $<6 \mathrm{~h} 23.7 \%$ and $>8 \mathrm{~h}$ $3.6 \%$. The usual bedtime was between 11 and $12 \mathrm{pm}$ (64.1\% of the sample), with $22.1 \%$ to sleep before $11 \mathrm{pm}$ and $13.8 \%$ after midnight. Wake-up time was between 6 and 7 am for $64.6 \%$ of the individuals, with $19 \%$ to wake up before $6 \mathrm{am}$ and $16.4 \%$ after $7 \mathrm{am}$. No awakenings during sleep were stated by $41 \%$ of individuals, whereas $27.7 \%$ woke up once every night and $20.5 \%$ twice. During weekdays $36.9 \%$ stated no naps and $29.2 \%$ reported taking one or two extra midday naps. Finally, during weekends, holidays and days off, $57.4 \%$ of the participants added extra bed hours $-44 \%$ up to 2 extra hours and $13.8 \%$ more than 2 hours.

From the total sample, 34/195 (17.4\%) worked in shifts. Symptoms of insomnia and fatigue were reported by $37.4 \%$ of the population, and sleepiness by $27 \%$.

\section{Prevalence and Characteristics of CRSWD}

Diagnostic criteria for CRSWD were fulfilled by 25 individuals (12.8\%) as follows: 10 for DSWPD (5.1\%), 13 for SWD (6.7\%), 1 for ISWRD (0.5\%), and 1 for ASWPD 
$(0.5 \%)$. No gender predominance was observed. CRSWD affected younger, but still middle-aged, individuals (mean age: $49.64 \pm 13.87 \mathrm{y}$ in CRSWD vs $55.68 \pm 13.06$ in nonCRSWD, $\mathrm{p}=0.033$ ). CRSWD patients' demographics and diagnostic workup are presented in Table 1.

A statistically significant difference was observed in usual bedtime between CRSWD group and general population $(12.10 \mathrm{pm} \pm 1.15$ vs $11.27 \mathrm{am} \pm 0.99$, respectively, $\mathrm{p}=0.002$ ) as expected by definition (Figure 1, Sleep Onset). No statistically significant difference was observed in wake-up time between the two groups, because of morning obligations (Figure 2, Sleep Offset). As a consequence, time in bed duration was significantly less in CRSWD when compared to non-CRSWD (6.5 $\pm 1.23 \mathrm{~h}$ vs $7.16 \pm 1.09 \mathrm{~h}$, respectively, $\mathrm{p}=0.017$ ), with more naps per week $(3.2 \pm 2.9$ vs $2.05 \pm 2.39$, respectively, $\mathrm{p}=0.031$ ) and more extra bed hours during days off, weekends and holidays $(1.56 \pm 1.07$ vs $1.08 \pm 1.32$, respectively, $\mathrm{p}=0.005$ ). Finally, CRSWDs' patients were statistically significantly more insomniac $(p=0.002)$, sleepy
( $p=0.002)$, and depressive $(p=0.001)$, when compared to the general population sample.

Specifically, in DSWPD patients' usual bedtime was delayed in comparison to normal individuals (1.2am \pm 0.59 vs $11.3 \mathrm{pm} \pm 0.99$, respectively, $\mathrm{p}<0.001)$. They also stated significantly less subjective sleep time $(5.55 \mathrm{~h} \pm 1.14$ vs 6.47 \pm 1.16 , respectively, $p=0.033$, Figure 3 , subjective sleep time $)$ and time in bed $(6 \mathrm{~h} \pm 1.22,7.16 \pm 1.09$, respectively, $\mathrm{p}=0.015$ ), with no significant difference concerning extra bed hours during days off, weekends and holidays or weekly naps, compared to the general population. They reported insomnia more often, according to AIS ( $\mathrm{p}=0.001)$, and depression, according to BDI-II $(\mathrm{p}<0.001)$, than normal subjects. No statistical difference was observed between the two groups in terms of age, ESS, FSS and HADS.

Finally, SWD patients were statistically younger in age (48.7 $\pm 9.4 y$ vs $55.7 \pm 13.06$, respectively, $p=0.024)$ and more prone to sleep as indicated by ESS (11.69 \pm 4.98 vs $7.31 \pm 5.13$, respectively, $\mathrm{p}=0.009$ ), than non-CRSWD

Table I CRSWD Patients' Demographics and Diagnostic Workup

\begin{tabular}{|c|c|c|c|c|c|c|c|c|c|c|c|}
\hline No & Age & $\begin{array}{c}\text { Gender } \\
\text { (M/F) }\end{array}$ & ESS & AIS & FSS & HAS & HDS & $\begin{array}{c}\text { BDI- } \\
\text { II }\end{array}$ & AHI & $\begin{array}{c}\text { Shifts } \\
\text { (No/Yes) }\end{array}$ & CRSWD \\
\hline I & 45 & $\mathrm{~F}$ & 18 & 5 & 3,56 & 14 & 13 & 15 & 12,3 & $\mathrm{~N}$ & DSWPD \\
\hline 2 & 45 & $\mathrm{~F}$ & 4 & 16 & 2,78 & 11 & 7 & 13 & 0,2 & $\mathrm{~N}$ & DSWPD \\
\hline 3 & 59 & $M$ & 12 & 11 & 6,44 & 14 & 8 & 17 & 13,3 & $N$ & DSWPD \\
\hline 4 & 30 & $M$ & 8 & 9 & 5,56 & 13 & 6 & 15 & 2,3 & $\mathrm{~N}$ & DSWPD \\
\hline 5 & 24 & $M$ & 12 & 12 & 4,89 & 12 & 7 & 14 & 9,5 & $\mathrm{~N}$ & DSWPD \\
\hline 6 & 73 & $M$ & 8 & 16 & 6,00 & 9 & 8 & 15 & 9,2 & $N$ & DSWPD \\
\hline 7 & 53 & $M$ & 12 & 16 & 3,56 & 6 & 8 & 12 & 0,9 & $\mathrm{~N}$ & DSWPD \\
\hline 8 & 56 & $M$ & 6 & 7 & 3,56 & 0 & 2 & 18 & 3,1 & $N$ & DSWPD \\
\hline 9 & 34 & $M$ & 9 & 9 & 3,56 & 12 & 9 & 21 & 5,5 & $N$ & DSWPD \\
\hline 10 & 67 & $\mathrm{~F}$ & 10 & 8 & 6,56 & 13 & 17 & 29 & 4 & $N$ & DSWPD \\
\hline II & 45 & $F$ & 18 & 4 & 3,56 & 11 & 8 & 8 & 7 & $Y$ & SWD \\
\hline 12 & 64 & $M$ & 11 & 7 & 5,00 & 6 & 3 & 10 & 11,4 & $Y$ & SWD \\
\hline 13 & 65 & $\mathrm{~F}$ & 17 & 2 & 4,22 & 6 & 2 & 5 & 14,3 & $Y$ & SWD \\
\hline 14 & 40 & $M$ & 4 & 7 & 3,44 & 10 & 9 & 14 & 1,6 & $Y$ & SWD \\
\hline 15 & 45 & $M$ & 9 & 6 & 4,00 & 3 & 0 & 4 & 3,7 & $Y$ & SWD \\
\hline 16 & 51 & $\mathrm{~F}$ & 10 & 12 & 6,67 & 12 & 10 & 9 & 3 & $Y$ & SWD \\
\hline 17 & 40 & $M$ & 10 & 9 & 4,22 & 10 & 9 & 13 & 2,7 & $Y$ & SWD \\
\hline 18 & 34 & $M$ & 11 & 5 & 4,67 & 6 & I & 4 & 8,2 & $Y$ & SWD \\
\hline 19 & 38 & $M$ & 11 & 5 & 4,00 & 0 & I & 6 & 4,3 & $Y$ & SWD \\
\hline 20 & 59 & $\mathrm{~F}$ & 20 & 8 & 5,56 & 10 & 10 & 5 & 12,5 & $Y$ & SWD \\
\hline 21 & 44 & $M$ & 13 & 16 & 4,00 & 3 & 4 & 8 & 13,6 & $Y$ & SWD \\
\hline 22 & 39 & $\mathrm{~F}$ & 3 & 7 & 4,33 & 8 & 6 & 10 & 2,4 & $Y$ & SWD \\
\hline 23 & 48 & $M$ & 15 & 4 & 5,00 & 2 & I & 4 & 1,5 & $Y$ & SWD \\
\hline 24 & 39 & $M$ & 15 & 4 & 4,22 & 12 & 10 & 0 & 12,4 & $\mathrm{~N}$ & ISWPD \\
\hline 25 & 83 & $\mathrm{~F}$ & 3 & 10 & 6,22 & 8 & 8 & 17 & 8 & $\mathrm{~N}$ & ASWPD \\
\hline
\end{tabular}




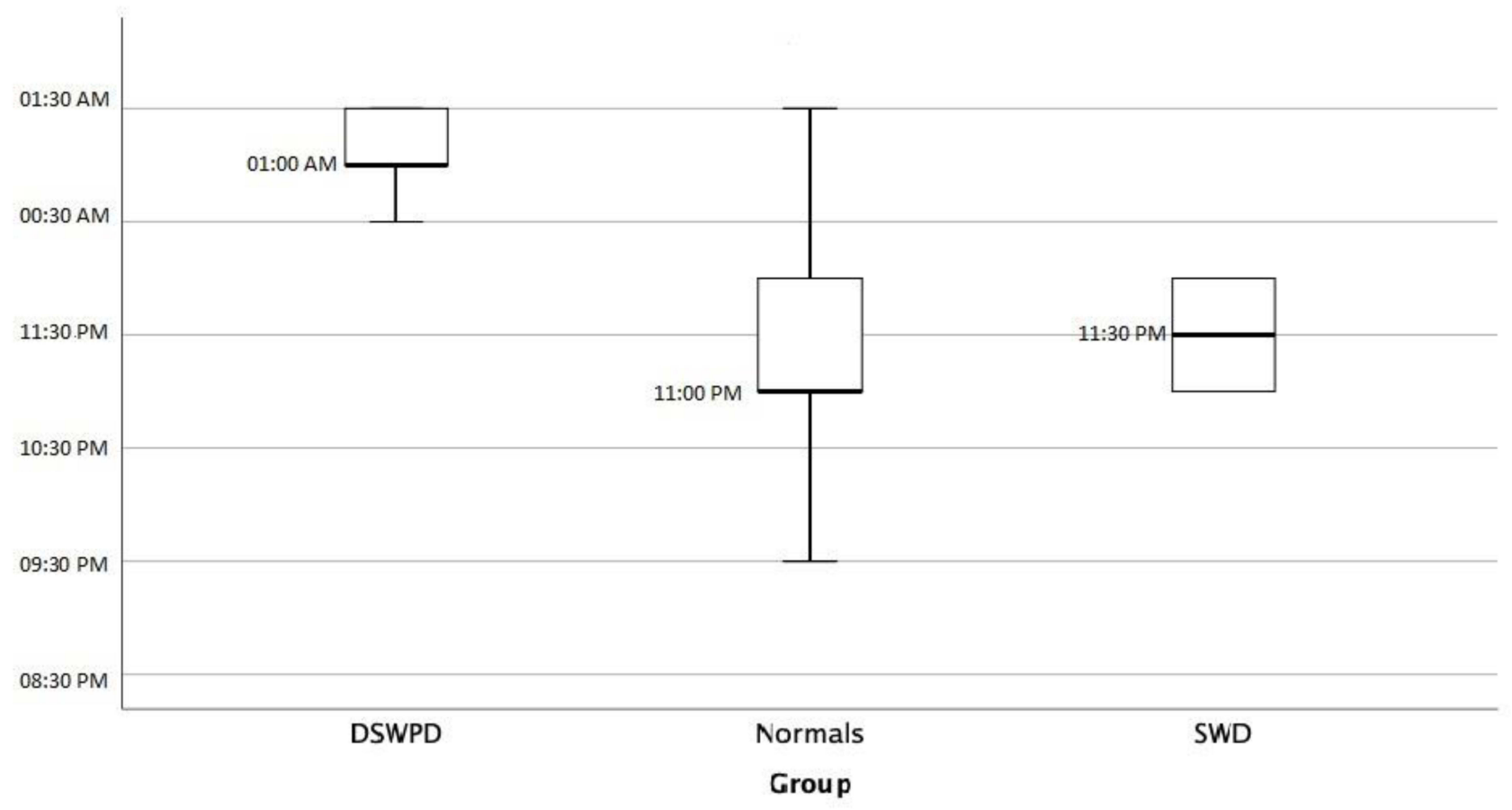

Figure I Sleep onset.

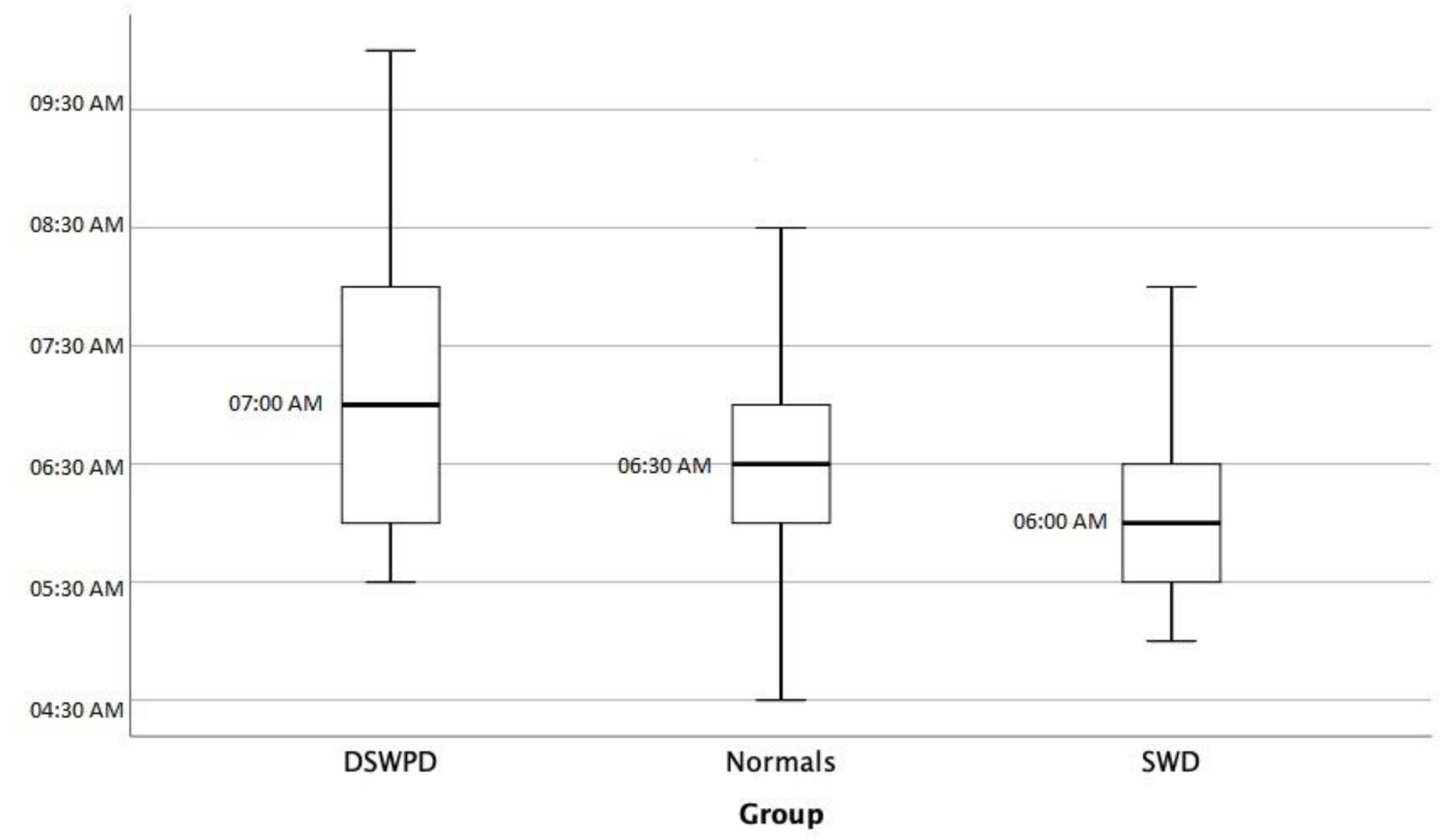

Figure 2 Sleep offset. 


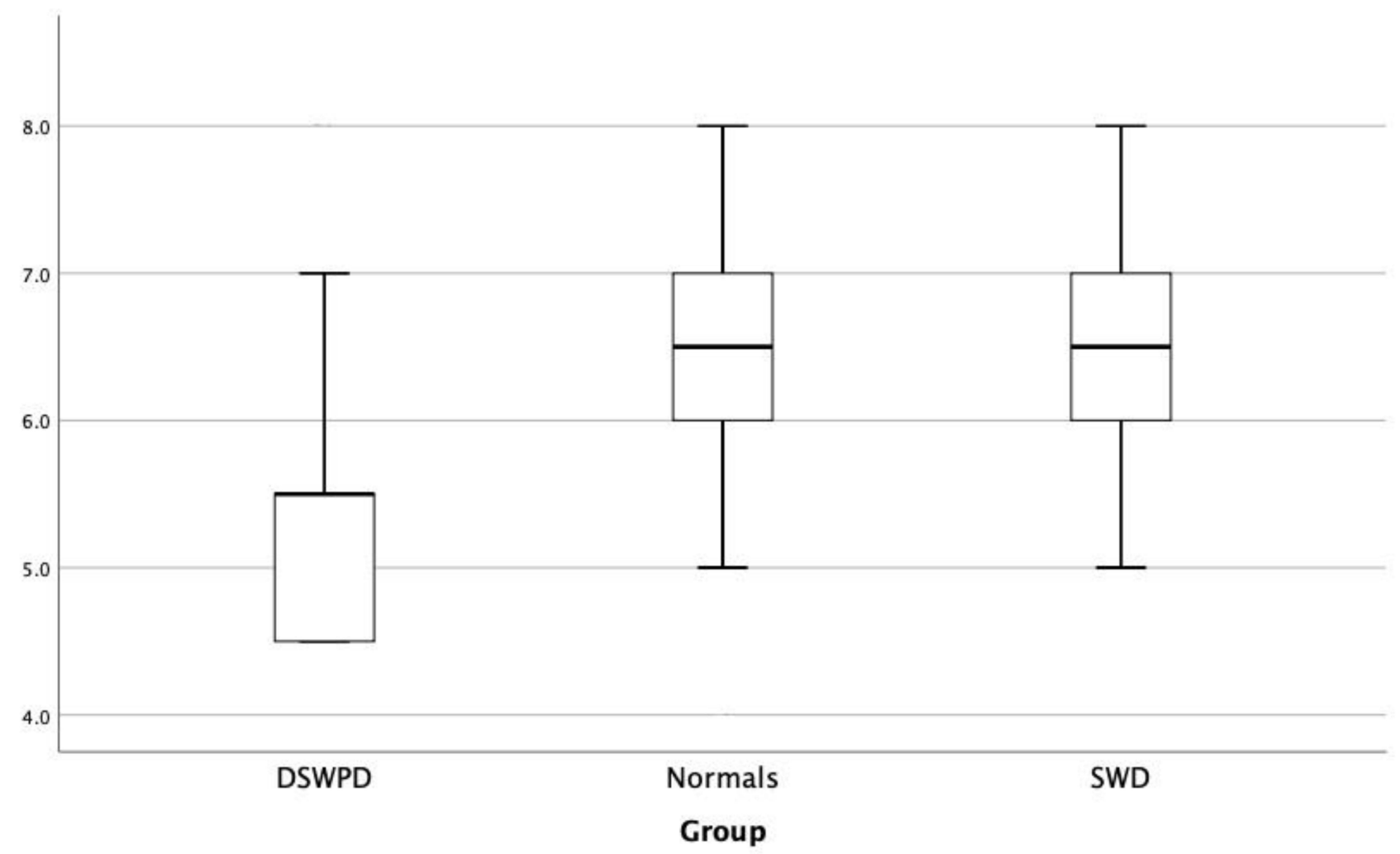

Figure 3 Subjective sleep time.

group. Sleep diary data for CRSWD patients are depicted in Table 2.

\section{Discussion}

To our knowledge, this is the only recent study assessing the prevalence of CRSWD in the general population, since the last was conducted two decades ago. According to our survey, adults in Cyprus, the southeast part of Europe, usually go to bed late and have reduced sleep duration, mainly because of a forced wake schedule for work, school, or other reasons. CRSWD is common in the general population, affecting $12.8 \%$ of individuals - especially DSWPD and SWD.

There is an increasing need to determine the normal patterns of sleep/wakefulness cycle and to establish the means for objective diagnosis of related symptoms and/or dysfunction of the circadian disorders. It is problematic to be determined on a global basis, considering geographic variations in light exposure and confounding factors (activities and common work schedule, cultural habits, etc.). Establishing specific quantitative diagnostic criteria for other sleep disorders, with obstructive sleep apnea (OSA), as the most profound example, enhanced progress in diagnostics, research and most importantly facilitated recognition and treatment. This major clinical impact can be partially attributed to a simple index (Apnea Hypopnea Index). ${ }^{17}$ Though oversimplification in not a panacea, the assumption that acceptable time cut-off points, according to the circadian cycle, are justified and can enable clinical diagnosis of CRSWD. In this content, a habitual sleep onset $\leq 9 \mathrm{pm}$ and sleep offset $\leq 5 \mathrm{am}$ were set as thresholds for the diagnosis of patients with ASWPD and falling asleep between 12am and 3am as a criterion for DSWPD.

Cyprus has an intense Mediterranean climate with an average of $9.8 \mathrm{~h}$ of daylight in December to $14.5 \mathrm{~h}$ in June. ${ }^{18}$ According to Cyprus Meteorology Department data, we calculated that yearly medium, sun rises at $5.35 \mathrm{am}$ and sets at $5.45 \mathrm{pm}$. Over the whole period of six months in the summer, there is an average of 11.5 hours of bright sunlight per day. It is well known that melatonin is suppressed by light and rises in the evening to reach a peak in the middle of the night. ${ }^{19}$ Melatonin is a "pacemaker" of circadian rhythm in humans promoting sleep propensity. Although endogenous circadian rhythms play a major role in the regulation of sleep and wakefulness, several external factors can also affect duration, 
Table 2 CRSWD Patients' Sleep Diary Data

\begin{tabular}{|c|c|c|c|c|c|c|c|}
\hline No & Sleep Onset & Sleep Offset & $\begin{array}{c}\text { Subj. Sleep } \\
\text { Time (h) }\end{array}$ & Time in Bed & Extra Bed Hours on Day Offs & Weekly Naps & CRSWD \\
\hline I & 1.00 & 6.30 & 5,5 & 5,5 & $\mathrm{I}, 5$ & 7 & DSWPD \\
\hline 2 & 1.30 & 9.30 & 5,0 & 8 & 1 & 0 & DSWPD \\
\hline 3 & 1.30 & 7.00 & 5,5 & 5,5 & 1,5 & 6 & DSWPD \\
\hline 4 & 2.30 & 10.00 & 7,0 & 7,5 & $\mathrm{I}, 5$ & 0 & DSWPD \\
\hline 5 & 1.30 & 7.00 & 5,5 & 5,5 & 2 & 1 & DSWPD \\
\hline 6 & 1.00 & 5.30 & 4,5 & 4,5 & 2 & 2 & DSWPD \\
\hline 7 & 00.30 & 5.30 & 4,5 & 5 & 1,5 & 6 & DSWPD \\
\hline 8 & 1.00 & 6.00 & 4,5 & 5 & 0 & 0 & DSWPD \\
\hline 9 & 00.30 & 8.00 & 8,0 & 7,5 & I,5 & I & DSWPD \\
\hline 10 & 01.00 & 7.00 & 5,5 & 6 & I,5 & 5 & DSWPD \\
\hline II & 23.00 & 8.00 & 8,0 & 9 & 0 & 0 & SWD \\
\hline 12 & 23.30 & 6.30 & 7,0 & 7 & 0 & 5 & SWD \\
\hline 13 & 23.00 & 6.00 & 7,0 & 7 & 0 & 7 & SWD \\
\hline 14 & 23.30 & 6.00 & 6,5 & 6,5 & 2,5 & 3 & SWD \\
\hline 15 & 23.00 & 5.30 & 6,0 & 6,5 & 3 & 2 & SWD \\
\hline 16 & 00.00 & 5.00 & 6,0 & 5 & 3,5 & 7 & SWD \\
\hline 17 & 00.00 & 6.00 & 6,0 & 6 & 2 & 0 & SWD \\
\hline 18 & 00.00 & 6.00 & 6,0 & 6,5 & 1,5 & 0 & SWD \\
\hline 19 & 23.00 & 6.00 & 7,0 & 7 & 2 & 5 & SWD \\
\hline 20 & 23.30 & 5.00 & 5,5 & 5,5 & 2 & 1 & SWD \\
\hline 21 & 00.00 & 7.00 & 5,0 & 7 & 0 & 3 & SWD \\
\hline 22 & 00.00 & 5.30 & 6,5 & 5,5 & 4 & 3 & SWD \\
\hline 23 & 00.00 & 8.00 & 7,0 & 8 & 1 & 5 & SWD \\
\hline 24 & 23.00 & 06.00 & 6,5 & 7 & 1 & 10 & ISWPD \\
\hline 25 & 21.00 & 06.00 & 6 & 9 & 2,5 & 1 & ASWPD \\
\hline
\end{tabular}

quality and appropriately timed sleep. $^{20}$ Occupation, family responsibilities, social and recreational opportunities, commute time and personal duties can lead to substantial divergences between the time and amount of sleep needed and the time and amount of sleep obtained. ${ }^{21}$ The most common work schedule in Cyprus is between $7.30 \mathrm{am}-3 \mathrm{pm}$ for the public sector and $8.00 \mathrm{am}-5 \mathrm{pm}$, with a lunch break interval for the private sector. Under normal working schedules, and not shift work, the decisive zeitgeber is the wake-up time and not the bedtime. Furthermore, a consensus statement exists about the minimum threshold value of 7 hours of sleep, as the appropriate amount in order to promote optimal health in adults. $^{22}$ Consequently, in order for an adult to achieve an adequate amount of sleep, the average bedtime is expected between 10.30 and $11.00 \mathrm{pm}$, and wake-up time between 6.30 and 7.00am.

\section{General Population}

In our study, the observed sleep/wake schedule in the great majority of participants differed from the "expected", in terms of sleep duration and bedtime. In accordance with our data, citizens of other Mediterranean countries, like Spain and Portugal, also report late bedtimes, possibly as a result of the climate conditions and the prolonged exposure to sunlight. ${ }^{23}$ Similarly, subjective sleep duration of $7-8 \mathrm{~h}$ per day report only an average of $31.4 \%$ (25.9$36.4 \%$ ) of individuals worldwide. ${ }^{23}$ The majority of the population suffers from sleep restriction during the week and usually takes midday naps or prolongs sleep during weekends, holidays and days off. Napping is common in all countries and not a specific habit in countries with high temperatures. $^{24}$

The $37.4 \%$ prevalence of AIS-defined insomnia in our population is consistent with data previously reported in similar surveys. ${ }^{23}$ Sleep deprivation and symptoms of insomnia probably cause daily dysfunction that can be expressed either as fatigue in FSS $(37.4 \%)$ or as sleepiness in ESS (27\%). Fatigue expresses tiredness and lack of energy without increased sleep propensity. Although it is a common complaint, it is underestimated, both in research projects and in daytime clinical work. 
Sleepiness expresses a subjective difficulty in maintaining wakefulness and an increased ease of falling asleep. Moderate to severe excessive daytime sleepiness is observed in about $10 \%$ of the general population according to a previous large epidemiological study. ${ }^{25}$ It is associated with socioeconomics (work, lifestyle, etc.), climate conditions (high temperatures) and a cardinal symptom in sleep clinics. ${ }^{23,26}$ Finally, HADS is a well validated and sensitive to change, screening instrument. Positive HADS-D expressed $8.2 \%$ and positive HADS-A expressed $17.4 \%$ of our participants. In the general population, the prevalence of depressive disorders is $7.8 \%$ and of anxiety disorders $14 \% .{ }^{27}$ Therefore, symptoms and dysfunction in our sample reflect similar prevalence as to previous studies and reassures the validity of the extracted results.

\section{CRSWD}

Primary CRSWDs result from an endogenous cause; however, a combination of innate phenomena and exogenous factors contribute to a varying degree. CRSWDs arise from a persistent pattern of sleep/wake disturbances, preventing affected individuals from going to sleep and awakening at "normal", desired times. As a result, affected individuals complain about symptoms of sleepiness and/or insomnia, functional impairment or depression. American Academy of Sleep Medicine (AASM) highlights patients' subjective concerns, instead of conventional and acceptable clock times, recognizing the relative nature of these terms. $^{7}$

In our study, we selected set time criteria for CRSWD, in relation to sunrise/sunset and the specified working hours in Cyprus. Actigraphy, dim melatonin secretion levels and nadir core temperature were not implemented in the study. As oriented by the ICD-3 diagnostic criteria, actigraphy would not add significantly in defining general cut-off hours. ${ }^{7}$

Dim melatonin levels are not routinely available and therefore diagnosis should be based on daily practice. We know that melatonin secretion normally begins around 9.30pm and ends around 7.30am. ${ }^{18}$ This is the timeframe for normal sleep.

The nadir core body temperature is about 2 hours before the habitual wake-up time or normally around $4.30 \mathrm{am} .{ }^{28}$ The use of medication, drugs, alcohol or shift work makes nadir clinical estimation very difficult. Therefore, 6.30am is generally accepted as a normal wakeup time.
Taking into account that usual required sleep offset in Cyprus is also 6.00-6.30am, then the desired sleep onset should be before $11.00-11.30 \mathrm{pm}$ for the minimum threshold value of 7 hours sleep. ${ }^{11}$

Considering all the above mentioned, setting as cut off points $9.00 \mathrm{pm}$, as the latest time to sleep, and $5.00 \mathrm{am}$ the latest time to wake up for ASWPD, and midnight past (6 hours after dark set), as the earliest hour to sleep for DSWPD, is probably an acceptable convention.

A significant proportion of our sample satisfied the diagnostic criteria for CRSWD (12.8\%). Affected individuals were usually middle-aged persons probably because of biological, social, professional and family reasons. No gender differences were statistically important, in accordance with previous reports. ${ }^{29,30}$

The key feature for many patients was chronically inadequate sleep. Insomnia and fatigue were the most common complaints, whereas sleepiness and depression/ anxiety were less common symptoms.

Previous studies, conducted a few decades before, estimated the prevalence of CRSWD between $0.13 \%$ and $0.17 \%$ in the general population. ${ }^{9,10}$ This huge difference can be attributed to several reasons. Firstly, both studies were conducted 20-30 years ago. Since then, lifestyle has changed, social pressures and obligations have increased, and most importantly technology and electrical devices which are actually artificial bright light sources, are interwoven with modern life. Secondly, there were methodological issues and low response rate in these pioneer studies. Finally, a serious issue when screening for CRSWD is that a validated and broadly used screening questionnaire is missing and therefore patients can be ignored and misinterpreted. To avoid confusion, the morningness-evenness questionnaire is not intended to screen for circadian rhythm disturbances. ${ }^{31}$

In the Japanese epidemiological study, ${ }^{9}$ two groups were formed serving no epidemiological cause as it was not a case/control study. Also, the first three initial screening questions were not specific and only partially covered the clinical picture of CRSWD and the second screening questionnaire used was not validated nor approved for this cause. Any other sleep disorder excluded the diagnosis of CRSWD a priori.

The Norwegian study ${ }^{10}$ had a good first response rate (77\%); however, the second response rate was very poor (28\%). Mailing was at the end of November, during the dark period in the region of Norway, north of the Arctic Circle (as the authors stated), when nights last 20 hours, 
and weeks pass without seeing sunlight. The sun is the regulator of the circadian rhythm thus we believe the study was conducted under extremely inappropriate conditions. Only 36 out of 129 suspected for DSWPD completed a sleep log; therefore, documented objective information was scarce in the study. Except for the 17 diagnosed as DSWPD, 54 subjects were classified as Motivated Sleep Phase Delay (MSPD) further reducing the prevalence. The investigators placed emphasis on separating individuals with DSWPD from those whom a delayed sleep phase was socially/environmentally or psychologically induced, and they realized that the standard ICSD criteria did not discriminate the two conditions satisfactorily. So, they added two additional criteria, inability to advance sleep period before $01.00 \mathrm{am}$ and sleep onset rarely occurring before 2.00am.

DSWPD is the most common disorder among CRSWD. It is considered prevalent in adolescents and young adults and unusual in older age, with a reported frequency in sleep disorder clinics of $6.7-16 \%{ }^{32}$ However, the number of cases of DSPWD seems to have increased in the last decades due to many aspects of modern life. We have to point out that our survey was conducted in the pre-Covid19 pandemic era as the restrictions, home quarantine and stress associated with the pandemic have a tremendous impact on sleep bio parameters. $^{33}$ According to our survey, DSWPD was present in 10 individuals (5.1\%). Our affected individuals were middle-aged, and they mainly complained about insomnia. Depression was the most common psychopathology associated with DSPD, in accordance with previous evidence. ${ }^{34}$ Though DSWPD patients have less time in bed, there is an absence of significant difference between DSWPD patients and the general population concerning the need for extra hours of sleep during days off, weekends and holidays or weekly naps. This is partially explained as the cardinal symptom of these patients is insomnia and not excessive daytime sleepiness. The propensity for sleep seems to be influenced by behaviour and the situation in which it is measured ${ }^{35}$ but can also reflect genetic differences in inherent sleep need. ${ }^{36}$ Furthermore, the duration of time in bed is a poor index for sleep deprivation as sleep efficiency and sleep quality is to be considered.

An estimated $20 \%$ of workers worldwide and $17.4 \%$ in our sample are involved in some form of shift work ${ }^{21}$ and that is why SWD is considered quite common; however, there are no reliable epidemiological studies available. The prevalence is unclear as there are individual differences in susceptibility to phase tolerance and adaptation. SWD was the most common disorder among CRSWD in our population $(6.7 \%)$. Sleepiness was the prevalent symptom in SWD patients.

Potential limitations should be acknowledged. The main drawback is that actigraphy and personal interviewing was not performed for verification. Logs provide longitudinal documentation of sleep-wake patterns, but actigraphy is recommended to ascertain a diagnosis. Questionnaires are only screening tools and cannot alone diagnose CRSWD but can objectively score symptoms and dysfunctions. Expert opinion is a necessity. Also, this is a cross-sectional study and the population is derived from a small segment of Southern Europe. The corresponding results are applicable only in Cyprus and are only indicative for the prevalence in Southern Europe.

Finally, we should also highlight that current diagnostic criteria do not facilitate a reliable diagnosis. A significant aspect of the normal circadian sleep rhythm is of course the adverse sunlight pattern. Sleep should follow dawn and end at sunrise. That is why southern countries are by nature late-phase sleepers while northerners tend to sleep earlier. This justifies in great extent our findings on DSWPD and supports the use of objective and less vague criteria for CRSWD. Epidemiological studies on CRSWD present several challenges in design and implementation to overcome potential biases and they are bound to fail if the criteria set for diagnosis are not accurate and objective.

As for the future prevalence of CRSWD, a momentous intervention is destined to apply in 2021. The European Parliament decided to discontinue the bi-annual clock changes throughout the European Union by $2021 .^{37}$

The yearly practice of setting the clocks one hour forward in March and one hour backward in November, for Daylight Saving Time purposes, can cause sleep problems as circadian rhythms are not aligned with natural cycles of light and darkness. Maybe this will have a positive effect on the circadian sleep-wake disturbances. Nevertheless, the major influencer, at least for DSWPD, seems to be the use of high-tech devices, ${ }^{38}$ operating like artificial light sources during the night, deregulating natural rhythms.

\section{Conclusions}

To our knowledge, this is the first study to describe the Southern European CRSWD prevalence. Emphasis was given on objective implements for assessment. 
Questionnaires for symptoms and dysfunction related to CRSWD as described in ICD-3 and pre-set sleep time limits. We conclude that the estimated prevalence for CRSWD in the general population of Cyprus is considerably high, affecting $12.8 \%$ of the general population. This is probably an era phenomenon due to lifestyle adjustments and technological advances. Further studies are required to generalize the increased global prevalence of CRSWD.

\section{Acknowledgments}

A sincere thank you to Ms Anna Sarris (Frederick University) for her constructive editing of the manuscript.

\section{Disclosure}

The authors report no conflicts of interest in this work.

\section{References}

1. Saeed Y, Zee PC, Abbott SM. The circadian clock is entrained to the environment through daily exposure to light and melatonin. Clinical neurophysiology of circadian rhythm sleep-wake disorders. Review Handb Clin Neurol. 2019;161:369-380. doi:10.1016/B978-0-44464142-7.00061-8

2. Reinberg A, Touitou Y, Restoin A, Migraine C, Levi F, Montagner H. The genetic background of circadian and ultradian rhythm patterns of 17-hydroxycorticosteroids: a cross-twin study. $J$ Endocrinol. 1985;105:247-253. doi:10.1677/joe.0.1050247

3. Sack RL, Auckley D, Auger RR, et al. Circadian rhythm sleep disorders: part I, basic principles, shift work and jet lag disorders. An American Academy of Sleep Medicine Review. Sleep. 2007;30 (11):1460-1483. doi:10.1093/sleep/30.11.1460

4. Sack RL, Auckley D, Auger RR, et al. Circadian rhythm sleep disorders: part II, advanced sleep phase disorder, delayed sleep phase disorder, free running disorder, and irregular sleep wake rhythm. An American Academy of Sleep Medicine Review. Sleep. 2007;30(11):1484-1501.

5. Weitzman ED, Czeisler CA, Coleman RM. Delayed sleep phase syndrome. Arch Gen Psychiatry. 1981;38:737-746. doi:10.1001/ archpsyc.1981.01780320017001

6. Thorpy MJ; chairman: Diagnostic Classification Steering Committee. International Classification of Sleep Disorders: Diagnostic and Coding Manual. Rochester, Minnesota: American Sleep Disorders Association; 1990.

7. International Classification of Sleep Disorders. 3rd ed. Darien, IL USA: American Academy of Sleep Medicine;2014.

8. Sateia MJ. International classification of sleep disorders-third edition: highlights and modifications. Chest. 146(5):1387-1394. doi:10.1378/ chest.14-0970

9. Yazaki M, Shirakawa S, Okawa M, Takahashi K. Demography of sleep disturbances associated with circadian rhythm disorders in Japan. Psychiatry Clin Neurosci. 1999;53(2):267-268. doi:10.1046/ j.1440-1819.1999.00533.x

10. Schrader H, Bovim G, Sand T. The prevalence of advanced and delayed sleep phase syndromes. J Sleep Res. 1993;2(1):51-55. doi:10.1111/j.1365-2869.1993.tb00061.x

11. Frangopoulos F, Nicolaou I, Zannetos S, et al. Estimating Obstructive Sleep Apnea in Cyprus: a randomized, stratified epidemiological study using STOP-BANG questionnaire. Sleep Med. 2019;61:37-43. doi:10.1016/j.sleep.2019.04.013
12. Johns MW. A new method for measuring daytime sleepiness: the Epworth Sleepiness Scale. Sleep. 1991;14(6):540-545. doi:10.1093/ sleep/14.6.540

13. Soldatos CR, Dikeos DG, Paparrigopoulos TJ. Athens Insomnia Scale: validation of an instrument based on ICD-10 criteria. $J$ Psychosom Res. 2000;48(6):555-560. doi:10.1016/S00223999(00)00095-7

14. Valko PO, Bassetti CL, Bloch KE, Held U, Baumann CR. Validation of the Fatigue Severity Scale in a Swiss cohort. Sleep. 16011607;2008(31).

15. Beck AT, Steer RA, Ball R, Ranieri W. Comparison of Beck Depression Inventories -IA and -II in psychiatric outpatients. J Pers Assess. 1996;67(3):588-597. doi:10.1207/s15327752jpa6703_13

16. Zigmond AS, Snaith RP. The hospital anxiety and depression scale. Acta Psychiatr Scand. 1983;67:361-370. doi:10.1111/j.16000447.1983.tb09716.x

17. Kapur VK, Auckley DH, Chowdhuri S, et al. Clinical practice guideline for diagnostic testing for adult obstructive sleep apnea: an American Academy of Sleep Medicine clinical practice guideline. J Clin Sleep Med. 2017;13(3):479-504. doi:10.5664/jcsm.6506

18. http://www.moa.gov.cy/moa/ms/ms.nsf/DMLcyclimate_en/ DMLcyclimate_en?OpenDocument.

19. Burgess HJ, Eastman CL. The dim light melatonin onset following fixed and free sleep schedules. J Sleep Res. 2005;14 (3):229-237.

20. Billings ME, Hale L, Johnson DA. CHEST Submission: contemporary Reviews in Sleep Medicine. The physical and social environment relationship with sleep health and disorders. Chest. 2020;157 (5):1304-1312. doi:10.1016/j.chest.2019.12.002

21. Basner M, Fomberstein KM, Razavi FM, et al. American time use survey: sleep time and its relationship to waking activities. Sleep. 2007;30:1085-1095. doi:10.1093/sleep/30.9.1085

22. Watson NF, Badr MS, Belenky G, et al. Joint Consensus Statement of the AASM and Sleep Research Society on the recommended amount of sleep for a healthy adult: methodology and discussion. J Clin Sleep Med. 2015;11(8):931-942. doi:10.5664/jcsm.4950

23. Soldatos CR, Allaert FA, Ohta T, Dikeos DG. How do individuals sleep around the world? Results from a single-day survey in ten countries. Sleep Med. 2005;6(1):5-13. doi:10.1016/j.sleep.2004.10.00

24. Dhand R, Harjyot Sohal H. Good sleep, bad sleep! The role of daytime naps in healthy adults. Curr Opin Pulm Med. 2006;12 (6):37982. doi:10.1097/01.mcp.0000245703.92311.d0

25. Bixler EO, Vgontzas AN, Lin HM, Calhoun SL, Vela-Bueno A, Kales A. Excessive daytime sleepiness in a general population sample: the role of sleep apnea, age, obesity, diabetes, and depression. J Clin Endocrinol Metab. 2005;90:4510-4515. doi:10.1210/jc.2005-0035

26. Economou NT, Ilias I, Velentza L, et al. Sleepiness, fatigue, anxiety and depression in Chronic Obstructive Pulmonary Disease and Obstructive Sleep Apnea - overlap - syndrome, before and after continuous positive airways pressure therapy. PLoS One. 2018;13 (6):e0197342. doi:10.1371/journal.pone.0197342

27. Wittchen HU, Jacobi F, Rehm J, et al. The size and burden of mental disorders and other disorders of the brain in Europe 2010. Eur Neuropsychopharmacol. 2011;21(9):655-679. doi:10.1016/j. euroneuro.2011.07.018

28. Wright H, Lack L, Bootzin R. Relationships between dim light melatonin onset and the timing of sleep in sleep onset insomniacs. Sleep Biolo Rhythms. 2006;4:78-80. doi:10.1111/j.14798425.2006.00194.x

29. Reis C, Paiva T. Delayed sleep-wake phase disorder in a clinical population: gender and sub-population differences. Sleep Sci. 2019;12(3):203-213. doi:10.5935/1984-0063.20190086

30. Drake CL, Roehrs T, Richardson G, et al. 2 shift work sleep disorder: prevalence and consequences beyond that of symptomatic day workers. SLEEP. 2004;27(8):1453-1462. doi:10.1093/sleep/ 27.8.1453 
31. Taillard J, Philip P, Chastang J-F, Bioulacn B. Validation of Horne and Ostberg morningness-eveningness questionnaire in a middle-aged population of French workers. $J$ Biol Rhythms. 2004;19(1):76-86. doi:10.1177/0748730403259849

32. Okawa M, Uchiyama M. Circadian rhythm sleep disorders: characteristics and entrainment pathology in delayed sleep phase and non-24 sleep-wake syndrome. Sleep Med Rev. 2007;11:485-496. doi:10.1016/j.smrv.2007.08.001

33. Salehinejad MA, Majidinezhad M, Ghanavati E, et al. Negative Impact of COVID-19 pandemic on sleep quantitative parameters, quality and circadian alignment: implications for health and psychological well-being. EXCLI J. 2020;19:1297-1308. doi:10.17179/ excli2020-2831

34. Regestein QR, Monk TH. Delayed sleep phase syndrome: a review of its clinical aspects. Am J Psychiatry. 1995;152:6028. doi:10.1176/ ajp.152.4.602
35. Johns MW. Sleep propensity varies with behaviour and the situation in which it is measured: the concept of somnificity. Sleep Res. 2002;11(1):61-67. doi:10.1046/j.1365-2869.2002.00274.x

36. Funato H, Miyoshi C, Fujiyama T, et al. Forward-genetics analysis of sleep in randomly mutagenized mice. Nature. 2016;539 (7629):378-383. doi:10.1038/nature20142

37. https://ec.europa.eu/transport/themes/summertime_en. Accessed June 11, 2021.

38. Ingram KK. Circadian rhythm sleep wake disorders (CRSWDs): linking circadian misalignment to adverse health outcomes. EBioMedicine. 2020;62:103142. doi:10.1016/j.ebiom.2020.103142

\section{Publish your work in this journal}

Nature and Science of Sleep is an international, peer-reviewed, open access journal covering all aspects of sleep science and sleep medicine, including the neurophysiology and functions of sleep, the genetics of sleep, sleep and society, biological rhythms, dreaming, sleep disorders and therapy, and strategies to optimize healthy sleep.
The manuscript management system is completely online and includes a very quick and fair peer-review system, which is all easy to use. Visit http://www.dovepress.com/testimonials.php to read real quotes from published authors. 